\title{
Local Molecular Reactivity of the Colored Dansylglycine in Water and Dioxane Studied through Conceptual DFT
}

\author{
Juan Frau' ${ }^{1}$ and Daniel Glossman-Mitnik $\mathbb{D}^{1,2}$ \\ ${ }^{1}$ Departament de Química, Universitat de les Illes Balears, Palma de Mallorca 07122, Spain \\ ${ }^{2}$ Laboratorio Virtual NANOCOSMOS, Departamento de Medio Ambiente y Energía, Centro de Investigación en Materiales \\ Avanzados, Miguel de Cervantes 120 Complejo Industrial Chihuahua, 31136 Chihuahua, CHIH, Mexico
}

Correspondence should be addressed to Daniel Glossman-Mitnik; daniel.glossman@cimav.edu.mx

Received 12 February 2018; Accepted 20 May 2018; Published 15 July 2018

Academic Editor: Philippe Dugourd

Copyright (c) 2018 Juan Frau and Daniel Glossman-Mitnik. This is an open access article distributed under the Creative Commons Attribution License, which permits unrestricted use, distribution, and reproduction in any medium, provided the original work is properly cited.

\begin{abstract}
This study evaluated a fixed long-range corrected range-separated hybrid (RSH) density functional associated with the Def2TZVP basis set alongside the SMD solvation model for the computation of the structure, molecular properties, and chemical reactivity of the M8 intermediate melanoidin pigment in the presence of water and dioxane. The preference of the active sites pertinent to radical, nucleophilic, and electrophilic attacks is made through linking them with the electrophilic and nucleophilic Parr functions, Fukui function indices, and condensed dual descriptor which are chemical reactivity descriptors that arise from conceptual density functional theory. The study confirmed the results from previous works showing that the MN12SX density functional is the most appropriate in predicting the chemical reactivity of this molecule in both solvents.
\end{abstract}

\section{Introduction}

Melanoidins are the final product of the Maillard reaction that starts with formation of a Schiff base between a reducing sugar and the amino group of a peptide or protein. Some of these molecules can be isolated, and it has been observed that they possess interesting properties that can be useful not only as dyes for coloring foods and for dye-sensitized solar cells (DSSC) but also for the design and development of drugs for the pharmaceutical industry.

One of these isolated molecules is called dansylglycine (or 2-[[5-(dimethylamino)naphthalen-1-yl]sulfonylamino] acetic acid) which has interesting properties as a colored molecule and as a fluorescent dye in water and dioxane, and we believe that it could be of interest to study their molecular reactivity by using the ideas of conceptual DFT [1-5], in the same way of our previous works [6-10].

Thus, in this computational study, we will assess a powerful density functional in calculating the molecular properties and structure of the dansylglycine pigment in the presence of water and dioxane as solvents. Following the same ideas of previous works, we will consider a fixed RSH functional instead of the optimally tuned RSH density functionals that have attained great success [11-18].

\section{Theoretical Background}

If we consider the KID (for Koopmans in DFT) procedure presented in our previous works [6-10] together with a finite difference approximation, then the global reactivity descriptors can be calculated starting from the orbital energies of the HOMO and LUMO: electronegativity $\chi[19,20]$, global hardness $\eta[19,20]$, electrophilicty $\omega[21]$, electrodonating $\omega^{-}$[22], electroaccepting power $\omega^{+}$[22], and net electrophilicity $\Delta \omega^{ \pm}$[23].

Applying the same ideas related to the KID procedure, the local reactivity descriptors can be expressed as follows: nucleophilic Fukui function: $f^{+}(\mathbf{r})=\rho_{N+1}(\mathbf{r})-\rho_{N}(\mathbf{r})$ [19], electrophilic Fukui function: $f^{-}(\mathbf{r})=\rho_{N}(\mathbf{r})-\rho_{N-1}(\mathbf{r})$ [19] dual descriptor: $\Delta f(\mathbf{r})=(\partial f(\mathbf{r}) / \partial N)_{v(\mathbf{r})}[24-30]$, nucleophilic Parr function: $P^{-}(\mathbf{r})=\rho_{s}^{\text {rc }}(\mathbf{r})[31,32]$, and electrophilic Parr function: $P^{+}(\mathbf{r})=\rho_{s}^{\text {ra }}(\mathbf{r})[31,32]$, where $\rho_{N+1}(\mathbf{r})$, 
TABLE 1: Global reactivity descriptors for the dansylglycine molecule calculated with the MN12SX/Def2TZVP model chemistry in water and in dioxane.

\begin{tabular}{lcccccc}
\hline & $\begin{array}{c}\text { Electronegativity } \\
(\alpha)\end{array}$ & $\begin{array}{c}\text { Chemical } \\
\text { hardness }(\eta)\end{array}$ & $\begin{array}{c}\text { Electrophilicity } \\
(\omega)\end{array}$ & $\begin{array}{c}\text { Electrodonating } \\
\text { power }\left(\omega^{-}\right)\end{array}$ & $\begin{array}{c}\text { Electroaccepting } \\
\text { power }\left(\omega^{+}\right)\end{array}$ & $\begin{array}{c}\text { Net electrophilicity } \\
\left(\Delta \omega^{ \pm}\right)\end{array}$ \\
\hline Water & 4.1950 & 3.6287 & 2.4248 & 4.2184 & 2.7183 & 6.9366 \\
Dioxane & 4.1427 & 3.6924 & 2.3240 & 4.0868 & 2.5974 & 6.6842 \\
\hline
\end{tabular}

TABLE 2: Maximum wavelength absorption $\left(\lambda_{\max }\right)$ of the dansylglycine molecule in water and dioxane calculated from the HOMO-LUMO gap and from TDDFT results in comparison with experimental value.

\begin{tabular}{lcccc}
\hline & $\lambda_{\max }(\mathrm{HL})$ & $\Delta(\mathrm{HL})$ & $\lambda_{\max }(\mathrm{TDDFT})$ & $\Delta(\mathrm{TDDFT})$ \\
\hline Water & 342 & 2 & 362 & 22 \\
Dioxane & 336 & 3 & 354 & 15 \\
\hline
\end{tabular}

$\rho_{N}(\mathbf{r})$, and $\rho_{N-1}(\mathbf{r})$ are the electronic densities at point $\mathbf{r}$ for the system with $N+1, N$, and $N-1$ electrons, respectively, and $\rho_{s}^{\mathrm{rc}}(\mathbf{r})$ and $\rho_{s}^{\mathrm{ra}}(\mathbf{r})$ are related to the atomic spin density (ASD) at the $\mathbf{r}$ atom of the radical cation or anion of a given molecule, respectively [33].

\section{Settings and Computational Methods}

Following the lines of our previous work [6-10], the computational studies were performed with the Gaussian 09 [34] series of programs with density functional methods as implemented in the computational package. The basis set used in this work was Def2SVP for geometry optimization and frequencies, while Def2TZVP was considered for the calculation of the electronic properties [35, 36]. All the calculations were performed in the presence of water and dioxane as solvents by doing integral equation formalismpolarized continuum model (IEF-PCM) computations according to the solvation model density (SMD) solvation model [37].

\section{Results and Discussion}

In the same way, as in our recent works on melanoidins [6-10], it has been found that the model chemistry formed by the connection between the MN12SX density functional and the Def2TZVP basis set is the best for justifying the fulfilling of the KID procedure for the dansylglycine molecule, both in the presence of water and dioxane. This, instead of presenting the comparison of the values of the orbital energies with the ionization potential I and the electron affinity A for different density functionals, we are showing the results for the global descriptors calculated with the MN12SX density functional in Table 1 comparing the values that arise from both solvents.

It is interesting to observe that the SMD solvation model is powerful enough to allow discriminating between the values for the global descriptors in both solvents.

In the past, various TDDFT studies of molecules of different sizes have used optimally tuned RSH density functionals with great success [11-18]. Table 2 provides a comparison between the results involved in the ground-state approximation derived from the HOMO-LUMO gap together with TDDFT results and the experimental value of $340 \mathrm{~nm}$ in water and $339 \mathrm{~nm}$ in dioxane. It is clearly observed from the results presented in tables that the MN12SX density functional in connection with the Def2TZVP basis set is capable of reproducing accurately the excitation energies of the dansylglycine molecule starting from the HOMO-LUMO gap in both solvents.

Having verified that the MN12SX/Def2TZVP model chemistry is a good choice for the calculation of the global reactivity descriptors and the prediction of the maximum absorption wavelength from the ground state calculation of the HOMO and LUMO, we now present the molecular structures of dansylglycine in water and in dioxane in Figure 1. Meanwhile, the calculated bond lengths and bond angles for both cases are shown in Tables S1-S4 of the Supplementary Information (SI).

The calculations of the condensed Fukui functions and dual descriptor are done by using the Chemcraft molecular analysis program to extract the Mulliken population analysis (MPA), natural population analysis (NPA), and Hirshfeld population analysis (HPA) atomic charges [38] beginning with single-point energy calculations involving the MN12SX density functional that uses the Def2TZVP basis set in line with the SMD solvation model and water and dioxane utilized as solvents.

Considering the potential application the dansylglycine molecule as an antioxidant, it is of interest to get insights into the active sites for radical attack. A graphical representation of the radical Fukui function $f^{0}$ calculated with the MN12SX/Def2TZVP model chemistry in water and dioxane is presented in Figure 2.

It can be concluded that the most favorable site for the radical attack will be N7 in both cases, being the chemical reactivity in water a bit larger than in dioxane.

A graphical representation of the dual descriptor $\Delta f_{k}$ of the dansylglycine molecule calculated with the MN12SX/Def2TZVP model chemistry in water and dioxane is presented in Figure 3.

It can be easily seen from the results for the dual descriptor $\Delta f(\mathbf{r})$ in Figure 3 that C13 will be the preferred site for a nucleophilic attack and that this atom will act as an electrophilic species in a chemical reaction. In turn, it can be appreciated that N6 will be more prone to electrophilic attacks and that these atomic sites will act as nucleophilic species in chemical reactions that involve the dansylglycine molecule in either solvent. As for the case of radical attacks, in these cases, the chemical reactivity in water will be larger 


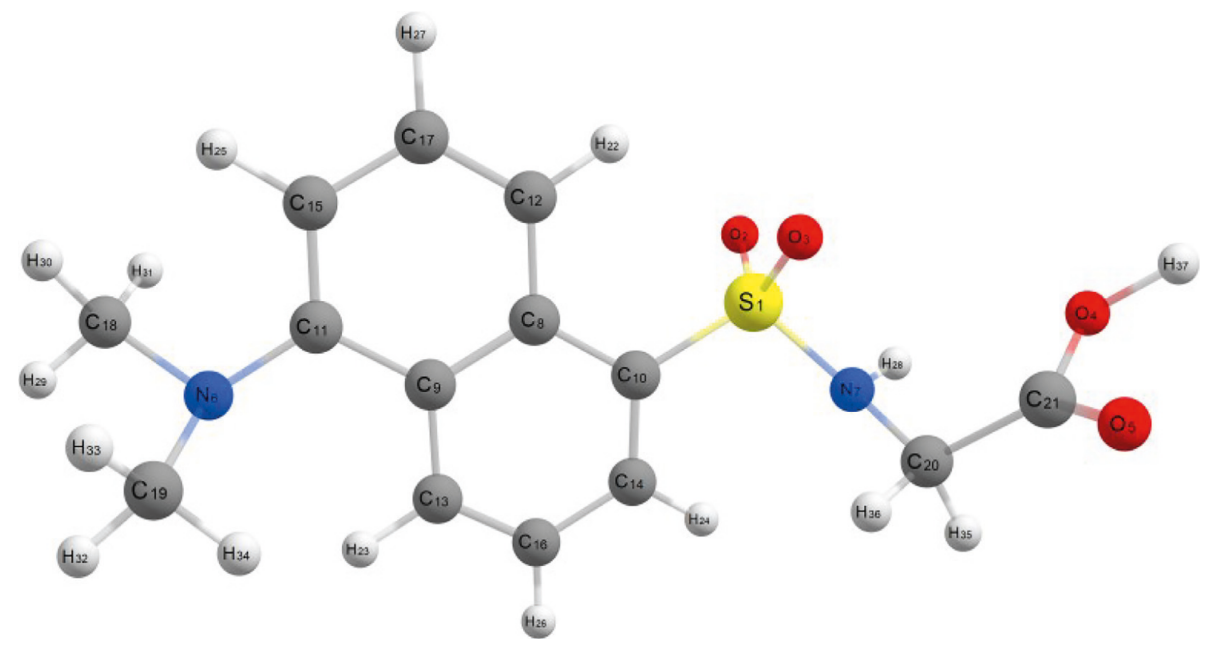

(a)

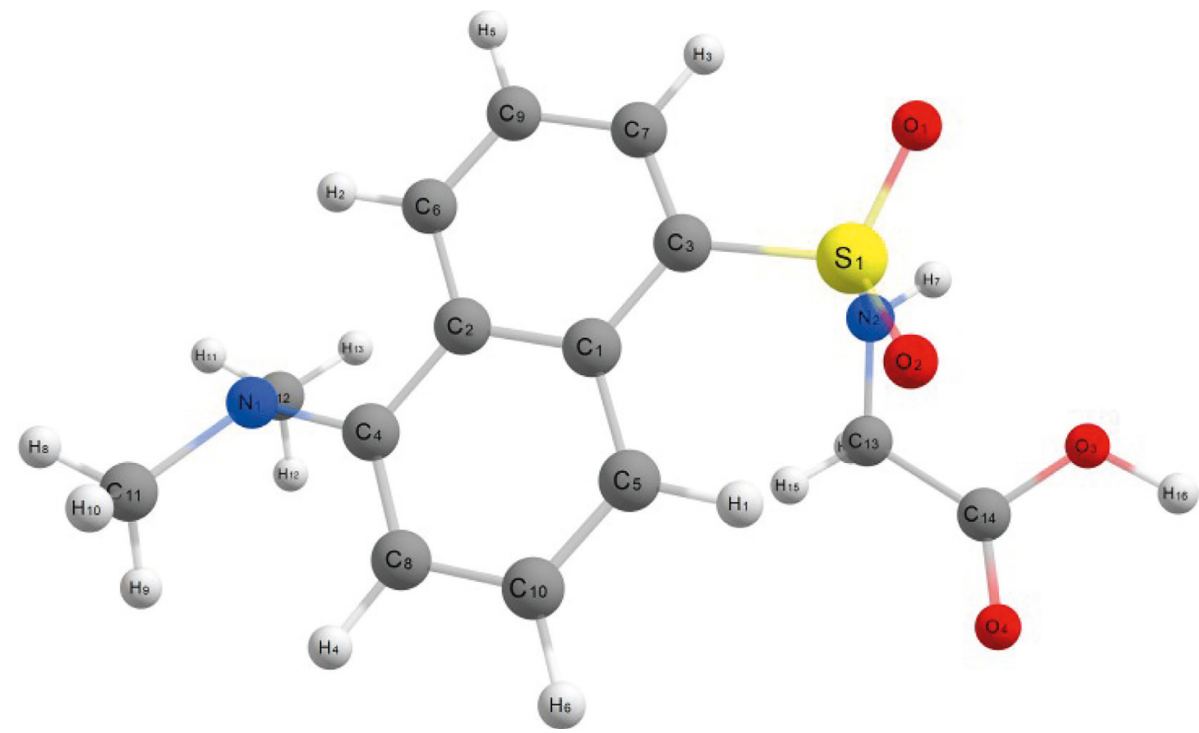

(b)

Figure 1: A schematic representation of the optimized structure of the dansylglycine molecule in water (a) and in dioxane (b) calculated with the MN12SX density functional showing the numbering of the atoms.

when considering dioxane as a solvent. The results for the condensed descriptor $\Delta f_{k}$ show the maximum positive value over $\mathrm{C} 13$ and the minimum negative value over $\mathrm{N} 6$ in both solvents using either population analysis.

Finally, the condensed electrophilic and nucleophilic Parr functions $P_{k}^{+}$and $P_{k}^{-}$over the atoms of the dansylglycine molecule in water and in dioxane have been calculated by extracting the Mulliken and Hirshfeld (or CM5) atomic charges using the Chemcraft molecular analysis program [38] starting from single-point energy calculations of the ionic species with the MN12SX density functional using the Def2TZVP basis set in the presence of the solvents according to the SMD solvation model. The maximum value of $P_{k}^{+}$is located over C13 ( 0.3616 for MPA and 0.2143 for HPA), and the maximum value of $P_{k}^{-}$is located over N6 (0.4804 for MPA and 0.3719 for HPA) for the calculation in the presence of water, while in dioxane the results are similar with $P_{k}^{+}=0.519$ for MPA and 0.2081 for HPA and $P_{k}^{-}=0.3814$ for MPA and 0.2962 for HPA. Thus, there is a nice agreement between the results describing the local reactivity of the dansylglycine molecule provided by Fukui functions, dual descriptor $\Delta f$ (or its condensed counterpart), and the Parr functions.

\section{Conclusions}

A fixed RSH density functional, namely, MN12SX, was examined to determine whether it fulfills the empirical KID procedure. The assessment was conducted by comparing the values from HOMO and LUMO calculations to those generated by the $\triangle \mathrm{SCF}$ technique for the dansylglycine molecule in water and in dioxane. This is a compound which is of academic as well as industrial interest. The study has confirmed that the range-separated hybrid meta-NGA density functional MN12SX is the most suited in meeting 


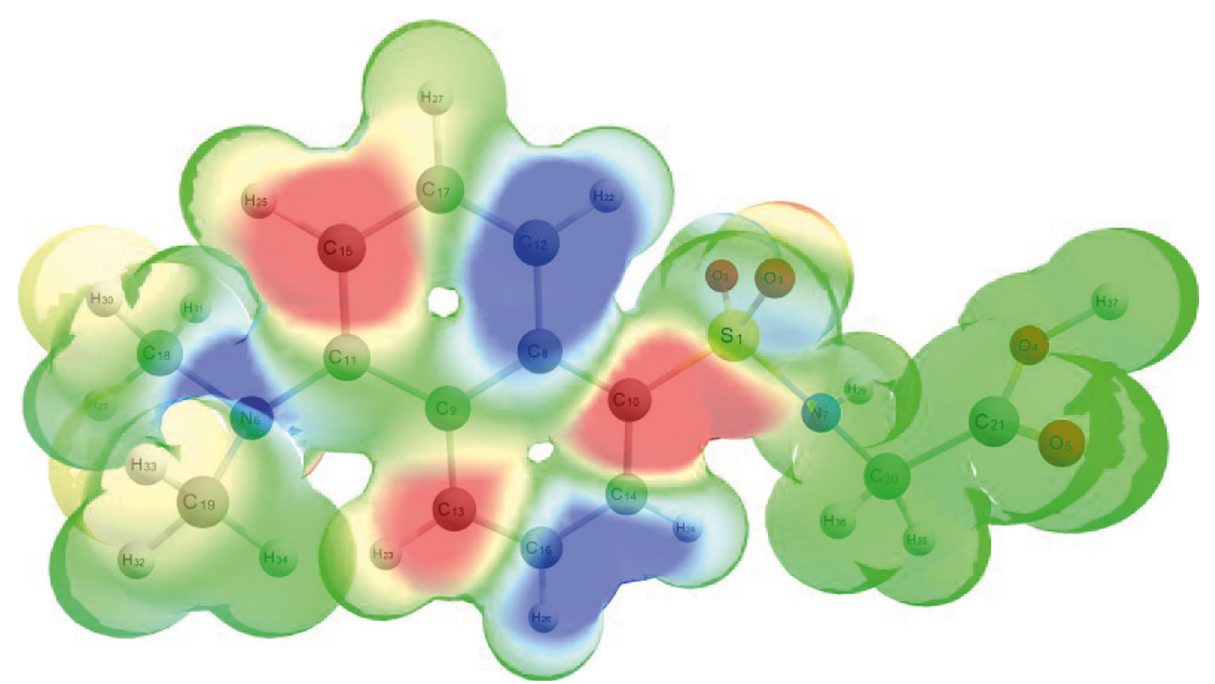

(a)

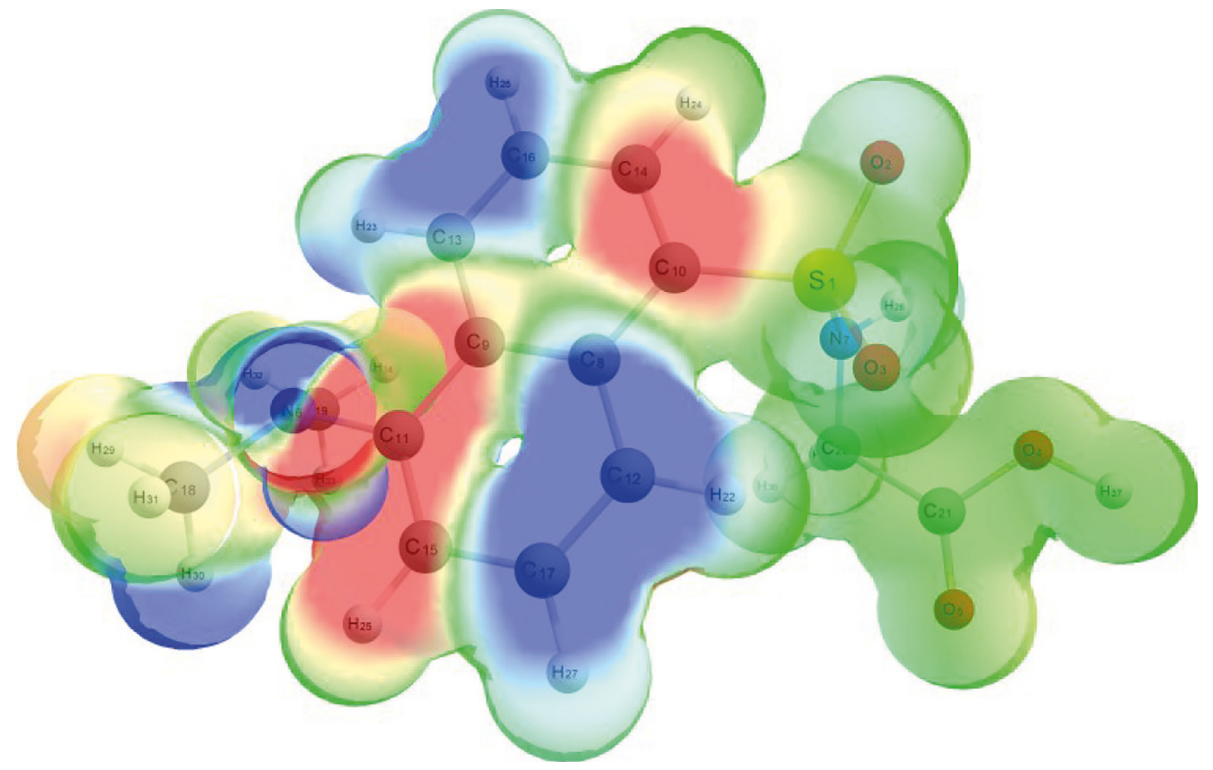

(b)

FIGURE 2: A graphical representation of the radical Fukui function $f^{0}$ of the dansylglycine molecule calculated with the MN12SX/Def2TZVP model chemistry in water (a) and dioxane (b).

this goal as it has been observed in previous works. Thus, this density functional can be a suitable alternative to density functionals where their behavior is optimally tuned using a gap-fitting procedure. It also exhibits the desirable prospect of benefiting future studies aimed at understanding the chemical reactivity of the colored products with larger molecular weights that are obtained when reducing sugars react with proteins and peptides.

From the results of this study, it can be concluded that the model chemistry formed by the connection of the MN12SX density functional and the Def2TZVP basis set is powerful enough to describe adequately the chemical reactivity of the dansylglycine molecule in both solvents through the calculation of the global and local DFT-based reactivity descriptors, including Fukui functions, Parr functions, and the dual descriptor calculations.

Furthermore, it is also possible to predict the maximum absorption wavelength for the dansylglycine in water an in dioxane with considerable accuracy. The prediction involves the MN12SX density functional beginning with the HOMO-LUMO gap instead of TDDFT calculations. Such a finding is particularly crucial considering the likelihood of it being used to inform the alternative determination method on the color that larger systems have such as prosthetic chromophore groups. This becomes necessary in circumstance when it is not possible to carry out TDDFT calculations as in the case of molecules of larger size like peptides and proteins. 


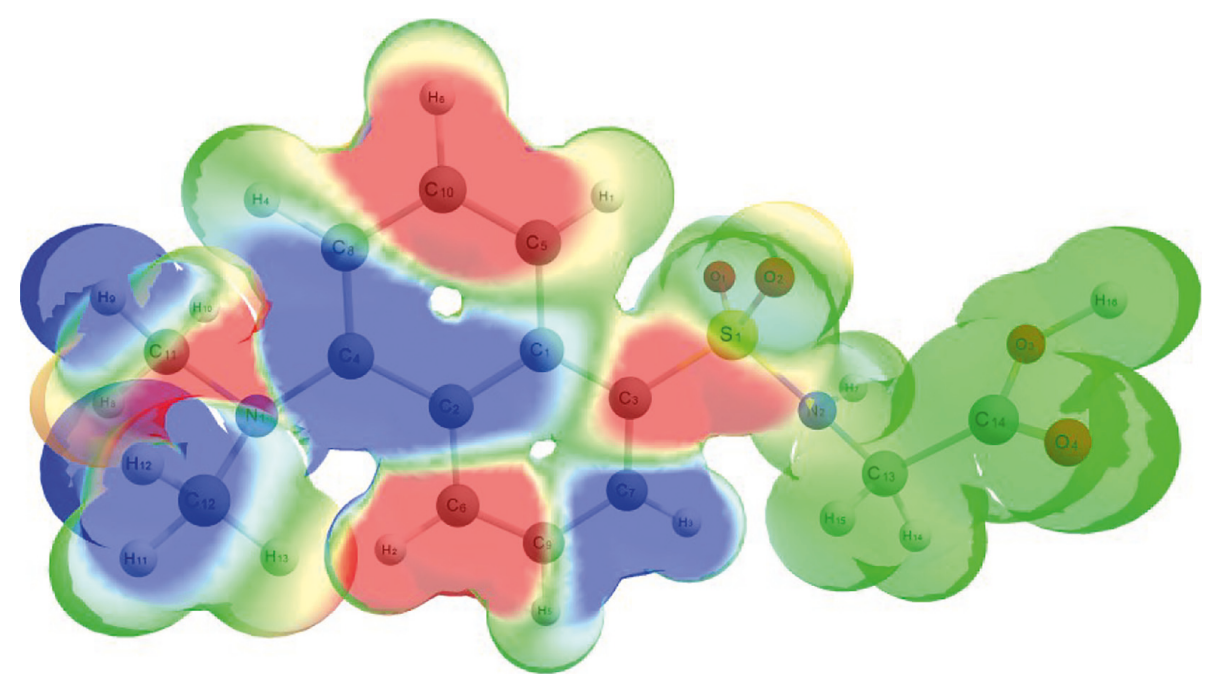

(a)

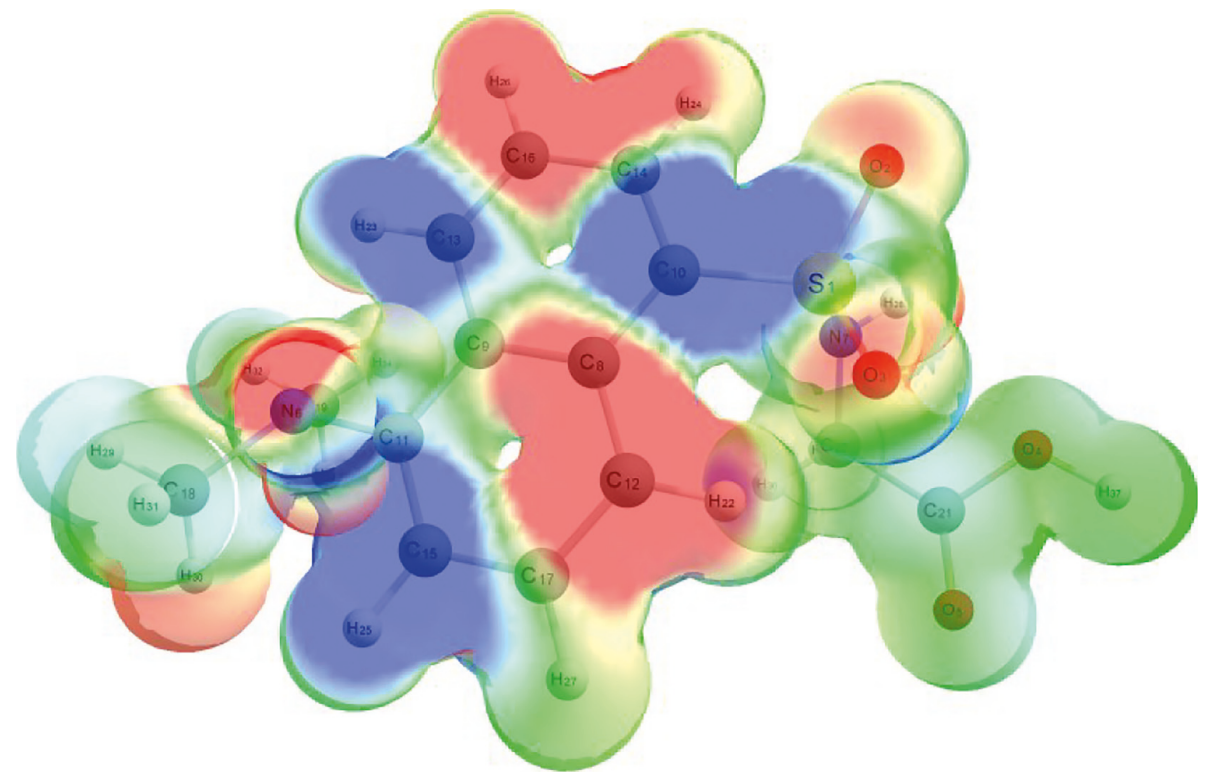

(b)

FIgURE 3: A graphical representation of the dual descriptor $\Delta f(\mathbf{r})$ of the dansylglycine molecule calculated with the MN12SX/Def2TZVP model chemistry in water (a) and dioxane (b).

\section{Data Availability}

All the underlying data related to this submission is available under request.

\section{Conflicts of Interest}

The received funding did not lead to any conflicts of interest regarding the publication of this manuscript, and there are no other conflicts of interest regarding this submitted paper.

\section{Acknowledgments}

This work has been partially supported by CIMAV, SC, and Consejo Nacional de Ciencia y Tecnología (CONACYT, Mexico) through Grant no. 219566/2014 for Basic Science
Research and Grant no. 265217/2016 for a Foreign Sabbatical Leave. Daniel Glossman-Mitnik conducted this work while a Sabbatical Fellow at the University of the Balearic Islands from which support is gratefully acknowledged. This work was cofunded by the Ministerio de Economía y Competitividad (MINECO) and the European Fund for Regional Development (FEDER) (CTQ2014-55835-R).

\section{Supplementary Materials}

Table S1: calculated bond lengths (in $\AA$ ) of the dansylglycine molecule with the MN12SX density functional using water as a solvent simulated with the SMD solvation model. Table S2: calculated bond angles (in ${ }^{\circ}$ ) of the dansylglycine molecule with the MN12SX density functional using water as a solvent simulated with the SMD solvation model. Table S3: 
calculated bond lengths (in $\AA$ ) of the dansylglycine molecule with the MN12SX density functional using dioxane as a solvent simulated with the SMD solvation model. Table S4: calculated bond angles (in ${ }^{\circ}$ ) of the dansylglycine molecule with the MN12SX density functional using dioxane as a solvent simulated with the SMD solvation model. (Supplementary Materials)

\section{References}

[1] R. Parr and W. Yang, Density-Functional Theory of Atoms and Molecules, Oxford University Press, Oxford, UK, 1989.

[2] T. Mineva, E. Sicilia, and N. Russo, "Density-functional approach to hardness evaluation and its use in the study of the maximum hardness principle," Journal of the American Chemical Society, vol. 120, no. 35, pp. 9053-9058, 1998.

[3] T. Mineva, N. Russo, and E. Sicilia, "Solvation effects on reaction profiles by the polarizable continuum model coupled with the gaussian density functional method," Journal of Computational Chemistry, vol. 19, no. 3, pp. 290-299, 1998.

[4] G. De Luca, E. Sicilia, N. Russo, and T. Mineva, "On the hardness evaluation in solvent for neutral and charged systems," Journal of the American Chemical Society, vol. 124, no. 7, pp. 1494-1499, 2002.

[5] E. Sicilia, N. Russo, and T. Mineva, "Correlation between energy, polarizability, and hardness profiles in the isomerization reaction of $\mathrm{HNO}$ and CINO," Journal of Physical Chemistry A, vol. 105, no. 2, pp. 442-450, 2001.

[6] J. Frau and D. Glossman-Mitnik, "Molecular reactivity and absorption properties of Melanoidin blue-G1 through conceptual DFT," Molecules, vol. 23, no. 3, pp. 559-615, 2018.

[7] J. Frau and D. Glossman-Mitnik, "Conceptual DFT study of the local chemical reactivity of the dilysyldipyrrolones A and B intermediate melanoidins," Theoretical Chemistry Accounts, vol. 137 , no. 5 , p. $1210,2018$.

[8] J. Frau and D. Glossman-Mitnik, "Conceptual DFT study of the local chemical reactivity of the colored BISARG melanoidin and its protonated derivative," Frontiers in Chemistry, vol. 6, no. 136, pp. 1-9, 2018.

[9] J. Frau and D. Glossman-Mitnik, "Molecular reactivity of some Maillard reaction products studied through conceptual DFT," Contemporary Chemistry, vol. 1, no. 1, pp. 1-14, 2018.

[10] J. Frau and D. Glossman-Mitnik, "Computational study of the chemical reactivity of the Blue-M1 intermediate melanoidin," Computational and Theoretical Chemistry, vol. 1134, pp. 22-29, 2018.

[11] A. Karolewski, T. Stein, R. Baer, and S. Kümmel, "Communication: tailoring the optical gap in light-harvesting molecules," Journal of Chemical Physics, vol. 134, no. 15, pp. 151101-151105, 2011.

[12] A. Karolewski, L. Kronik, and S. Kümmel, "Using optimally tuned range separated hybrid functionals in ground-state calculations: consequences and caveats," Journal of Chemical Physics, vol. 138, no. 20, article 204115, 2013.

[13] J. V. Koppen, M. Hapka, M. M. Szczeniak, and G. Chalasinski, "Optical absorption spectra of gold clusters $\mathrm{Au}(\mathrm{n})(\mathrm{n}=4,6$, $8,12,20)$ from long-range corrected functionals with optimal tuning," Journal of Chemical Physics, vol. 137, no. 11, article 114302, 2012.

[14] L. Kronik, T. Stein, S. Refaely-Abramson, and R. Baer, "Excitation gaps of finite-sized systems from optimally tuned range-separated hybrid functionals," Journal of Chemical Theory and Computation, vol. 8, no. 5, pp. 1515-1531, 2012.
[15] S. Refaely-Abramson, R. Baer, and L. Kronik, "Fundamental and excitation gaps in molecules of relevance for organic photovoltaics from an optimally tuned range-separated hybrid functional," Physical Review B, vol. 84, no. 7, pp. 075144-075148, 2011.

[16] T. Stein, L. Kronik, and R. Baer, "Prediction of charge-transfer excitations in coumarin-based dyes using a range-separated functional tuned from first principles," Journal of Chemical Physics, vol. 131, no. 24, article 244119, 2009.

[17] T. Stein, L. Kronik, and R. Baer, "Reliable prediction of charge transfer excitations in molecular complexes using timedependent density functional theory," Journal of the American Chemical Society, vol. 131, no. 8, pp. 2818-2820, 2009.

[18] H. Sun and J. Autschbach, "Electronic energy gaps for $\pi$-conjugated oligomers and polymers calculated with density functional theory," Journal of Chemical Theory and Computation, vol. 10, no. 3, pp. 1035-1047, 2014.

[19] R. Parr and W. Yang, "Density functional approach to the frontier-electron theory of chemical reactivity," Journal of the American Chemical Society, vol. 106, no. 14, pp. 4049-4050, 1984.

[20] P. Geerlings, F. De Proft, and W. Langenaeker, "Conceptual density functional theory," Chemical Reviews, vol. 103, no. 5, pp. 1793-1873, 2003.

[21] R. Parr, L. Szentpaly, and S. Liu, "Electrophilicity index," Journal of the American Chemical Society, vol. 121, no. 9, pp. 1922-1924, 1999.

[22] J. Gázquez, A. Cedillo, and A. Vela, "Electrodonating and electroaccepting powers," Journal of Physical Chemistry A, vol. 111, no. 10, pp. 1966-1970, 2007.

[23] P. Chattaraj, A. Chakraborty, and S. Giri, "Net electrophilicity," Journal of Physical Chemistry A, vol. 113, no. 37, pp. 10068-10074, 2009.

[24] C. Morell, A. Grand, and A. Toro-Labbé, "New dual descriptor for chemical reactivity," Journal of Physical Chemistry A, vol. 109, no. 1, pp. 205-212, 2005.

[25] C. Morell, A. Grand, and A. Toro-Labbé, “Theoretical support for using the descriptor," Chemical Physics Letters, vol. 425, no. 4-6, pp. 342-346, 2006.

[26] C. Cárdenas, N. Rabi, P. Ayers, C. Morell, P. Jaramillo, and P. Fuentealba, "Chemical reactivity descriptors for ambiphilic reagents: dual descriptor, local hypersoftness, and electrostatic potential," Journal of Physical Chemistry A, vol. 113, no. 30, pp. 8660-8667, 2009.

[27] A. Toro-Labbé, Theoretical Aspects of Chemical Reactivity, Elsevier Science, Amsterdam, Netherlands, 2007.

[28] P. Ayers, C. Morell, F. De Proft, and P. Geerlings, "Understanding the Woodward-Hoffmann rules by using changes in electron density," Chemistry-A European Journal, vol. 13, no. 29, pp. 8240-8247, 2007.

[29] C. Morell, P. Ayers, A. Grand, S. Gutiérrez-Oliva, and A. ToroLabbé, "Rationalization of the Diels-Alder reactions through the use of the dual reactivity descriptor," Physical Chemistry Chemical Physics, vol. 10, no. 48, pp. 7239-7246, 2008.

[30] C. Morell, A. Hocquet, A. Grand, and B. Jamart-Grégoire, “A conceptual DFT Study of hydrazino peptides: assessment of the nucleophilicity of the nitrogen atoms by means of the dual descriptor $\Delta f(\mathbf{r})$," Journal of Molecular Structure, vol. 849, no. $1-3$, pp. 46-51, 2008.

[31] L. R. Domingo, P. Pérez, and J. Sáez, "Understanding the local reactivity in polar organic reactions through electrophilic and nucleophilic Parr functions," RSC Advances, vol. 3, no. 5, pp. 1486-1494, 2013.

[32] E. Chamorro, P. Pérez, and L. R. Domingo, "On the nature of Parr functions to predict the most reactive sites along organic 
polar reactions," Chemical Physics Letters, vol. 582, pp. 141143, 2013.

[33] L. R. Domingo, M. Ríos-Gutiérrez, and P. Pérez, "Applications of the conceptual density functional theory indices to organic chemistry reactivity," Molecules, vol. 21, no. 6, p. 748, 2016.

[34] M. J. Frisch, G. W. Trucks, H. B. Schlegel et al., Gaussian 09 Revision D.01, Gaussian Inc., Wallingford, CT, USA, 2018.

[35] F. Weigend and R. Ahlrichs, "Balanced basis sets of split valence, triple zeta valence and quadruple zeta valence quality for $\mathrm{H}$ to $\mathrm{Rn}$ : design and assessment of accuracy," Physical Chemistry Chemical Physics, vol. 7, no. 18, pp. 3297-3305, 2005.

[36] F. Weigend, "Accurate Coulomb-fitting basis sets for H to R," Physical Chemistry Chemical Physics, vol. 8, no. 9, pp. 10571065, 2006.

[37] A. Marenich, C. Cramer, and D. Truhlar, "Universal solvation model based on solute electron density and a continuum model of the solvent defined by the bulk dielectric constant and atomic surface tensions," Journal of Physical Chemistry B, vol. 113, no. 8, pp. 6378-6396, 2009.

[38] G. A. Zhurko, Chemcraft: Graphical Program for Visualization of Quantum Chemistry Computations, Russian Federation, Ivanovo, Russia, https://chemcraftprog.com. 

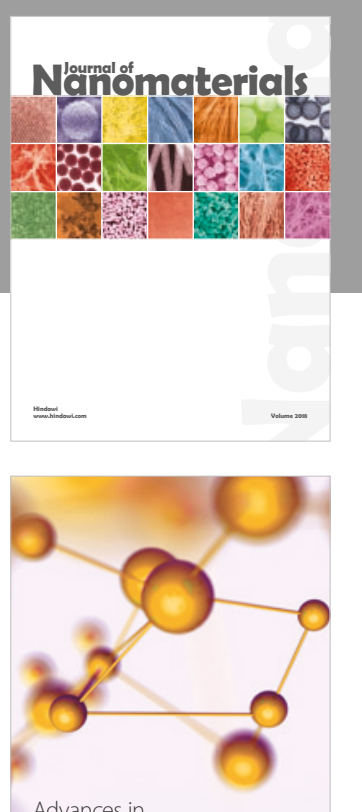

Physical Chemistry
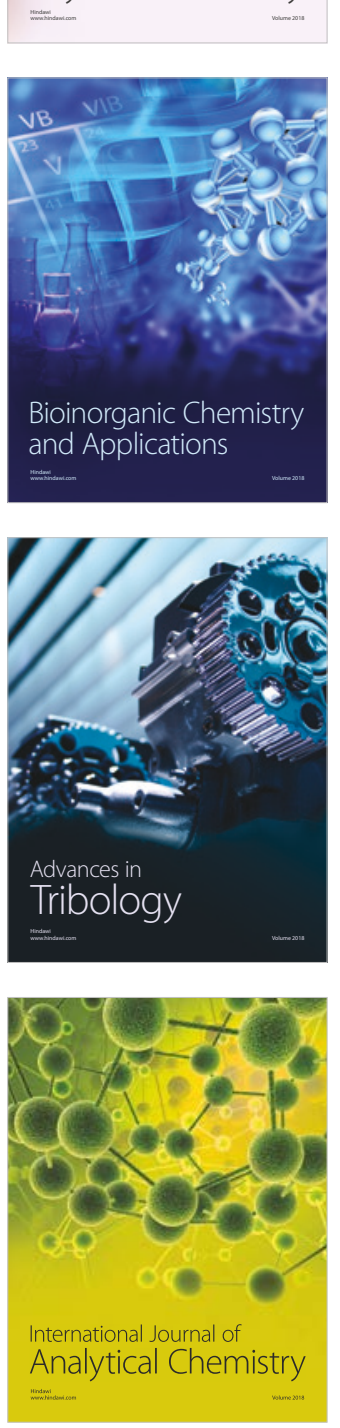

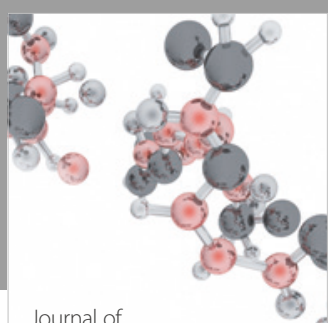

Analytical Methods

in Chemistry

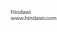

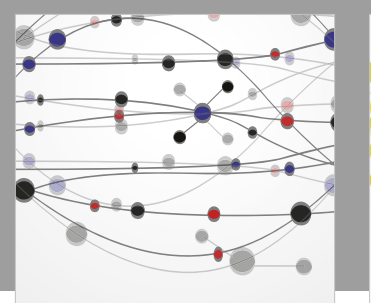

The Scientific World Journal

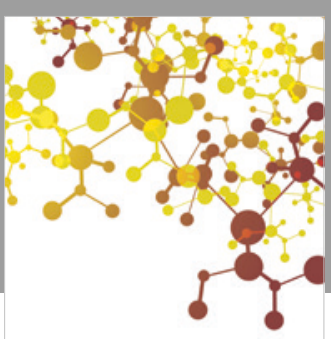

Journal of

Applied Chemistry
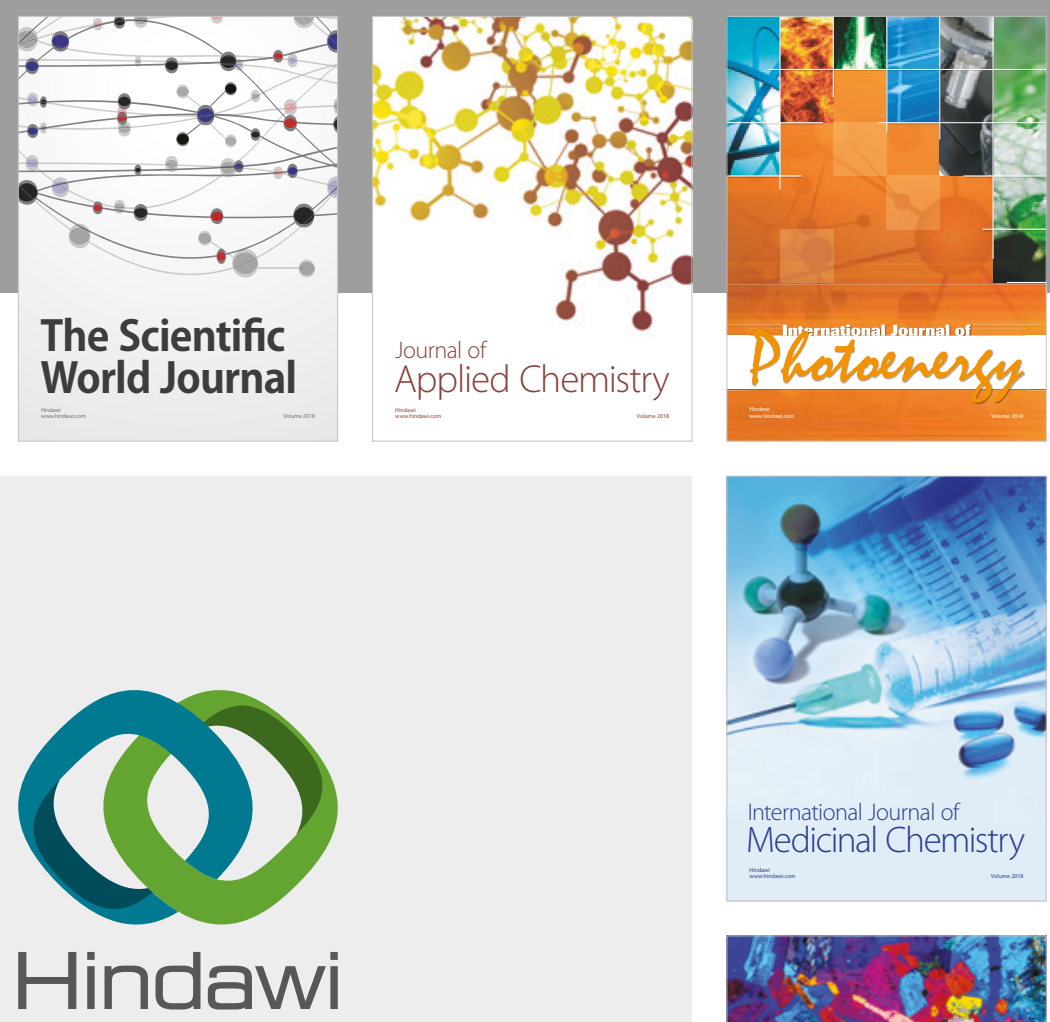

Submit your manuscripts at

www.hindawi.com
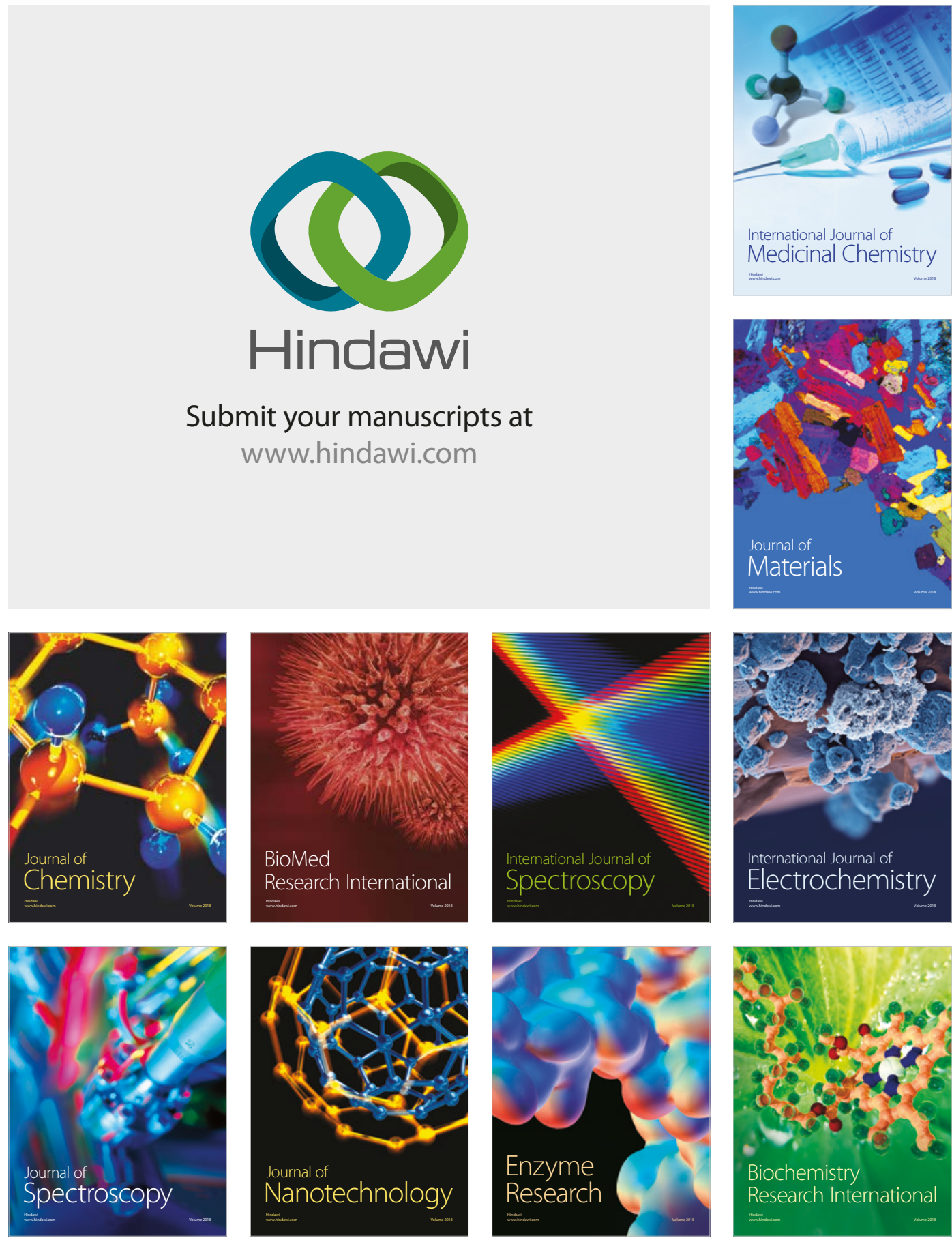
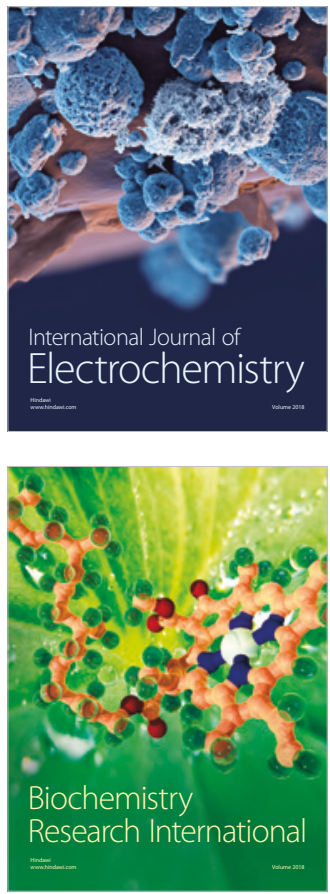